Vol. 8, n² | 2004

Varia

\title{
Avouer au seuil du gibet : enjeu social et judiciaire du testament de mort d'un brigand pendu à Genève en 1787
}

\section{Emanuel Gogniat}

\author{
(2) OpenEdition \\ Journals \\ Édition électronique \\ URL : https://journals.openedition.org/chs/463 \\ DOI : $10.4000 /$ chs.463 \\ ISSN : 1663-4837 \\ Éditeur \\ Librairie Droz

\section{Édition imprimée} \\ Date de publication : 1 novembre 2004 \\ Pagination : 63-84 \\ ISBN : 2-600-00803-5 \\ ISSN : 1422-0857
}

Référence électronique

Emanuel Gogniat, « Avouer au seuil du gibet : enjeu social et judiciaire du testament de mort d'un brigand pendu à Genève en 1787 », Crime, Histoire \& Sociétés / Crime, History \& Societies [En ligne], Vol. 8, n² | 2004, mis en ligne le 26 février 2009, consulté le 12 avril 2022. URL : http:// journals.openedition.org/chs/463 ; DOI : https://doi.org/10.4000/chs.463

Ce document a été généré automatiquement le 12 avril 2022.

(C) Droz 


\title{
Avouer au seuil du gibet : enjeu social et judiciaire du testament de mort d'un brigand pendu à Genève en $1787^{1}$
}

\author{
Emanuel Gogniat
}

1 À l'époque moderne, la criminalité associative représente l'une des inquiétudes majeures de la justice pénale européenne : le glaive s'oppose à la bande qui ajoute à la violence de sang le crime contre les biens, la mobilité géographique et les complicités étendues ${ }^{2}$. L'existence des « testaments de mort » dans les archives illustre bien l'effort consacré à sa répression. Selon la doctrine, le «testament de mort » désigne un texte qui contient les dernières déclarations d'un condamné à mort. Celles-ci sont notées par un juge, soit en prison, soit sur les lieux du supplice. Voulant peut-être soulager sa conscience avant de mourir, le condamné révèle des faits, jusque-là niés. Il espère parfois échapper au châtiment. Dans la procédure inquisitoire, les aveux fondent la preuve du crime et motivent la sentence. Rendus publics, ils peuvent en outre charger les complices dénoncés ${ }^{3}$. Comme on va le lire, le «cas exemplaire » de François Rosay illustre parfaitement la pratique du «testament de mort», répandue dans toute l'Europe, mais encore peu étudiée par les historiens de la justice pénale sous l'Ancien Régime ${ }^{4}$.

2 Le 14 juin 1787, dans la République de Genève, encadré de soldats, le « bandit » François Rosay gravit les marches du gibet dressé aux portes de la ville. Bientôt, face à la foule réunie à son de trompe, la corde du bourreau brise la nuque du jeune homme. Avant d'être ainsi étranglé, il ne lui reste guère de temps pour «soulager sa conscience du poids de ses crimes ». Dans ce but, les trois magistrats qui l'accompagnent l'exhortent à confirmer ses aveux consignés dans un «testament de mort». Inséré dans l'énorme procès intenté contre Rosay pour " criminalité associative », ce document se compose de vingt-sept pages manuscrites ${ }^{5}$. La singulière ampleur de ce «testament de mort » peu commun mérite qu'on le lise, notamment en raison de la manière dont il illustre la 
dimension sociale de la criminalité associative à l'époque moderne. Si ce " testament de mort » documente les pratiques pénales liées à la politique du gibet sous l'Ancien Régime, il permet en outre de reconstruire la biographie de l'homo criminalis, ici un bandit accusé de «rixes », "vols» et «assassinats ». Marquée par le «testament de mort ", la biographie judiciaire met en évidence «l'endurcissement criminel de Rosay ». De plus, une cinquantaine de témoins mandés en justice évoquent de manière unanime la "dangerosité » sociale de Rosay, notamment sa brutalité, sa connaissance des armes, ses complicités, sa mobilité et ses activités nocturnes. Selon les témoins à charge, le jeune bandit est parfaitement inséré dans les réseaux et les lieux de la sociabilité traditionnelle : cabarets, foires, routes.

Basé sur la procédure inquisitoire depuis le $\mathrm{XVI}^{\mathrm{e}}$ siècle, le système pénal genevois ne compte qu'un seul niveau de juridiction en matière de grande criminalité ${ }^{\text {. }}$ L'information commence soit par une plainte, une dénonciation ou sur instance du parquet (procureur général). L'instruction terminée, soit lorsque les auditeurs de justice ont interrogé le prévenu, auditionné les témoins, cherché d'éventuelles preuves matérielles et demandé des expertises (chirurgiens, médecins, serruriers, charpentiers et autres spécialistes professionnels) pour établir les «circonstances » matérielles du crime, les pièces judiciaires, numérotées et inventoriées, reviennent au procureur général. Le magistrat les utilise pour forger son réquisitoire - «Conclusions »-qui qualifie le crime, motive la peine et exige son application. À la modernité judiciaire du parquet s'ajoute en outre celle de la défense : dès 1734, les inculpés de Genève ont le droit d'être défendus par un avocat durant le procès devant le Petit Conseil de la République, autorité politique et juge suprême ${ }^{7}$. Finalement, dès 1738 , la "question préalable» est abolie. En l'absence d'un Code pénal fixant le délit à la peine, les magistrats du Petit Conseil prononcent leur sentence en se basant sur les «Conclusions » du procureur général motivées par la doctrine pénale, la jurisprudence et le droit romain. Juridiquement sans limite, leur pouvoir judiciaire est ainsi limité par le réquisitoire du procureur général. Le procès de François Rosay s'insère dans ce modèle pénal.

\section{François Rosay, Hercule du crime}

Né le 21 janvier 1766 à Lugrin, petite paroisse savoyarde proche d'Évian, Rosay est le fils d'un pauvre cordonnier. Comptant déjà quelques petits larcins à son actif, il quitte la maison pour s'engager dans le régiment de Savoie en compagnie de son frère. Il déserte au bout de quelques semaines et revient dans sa région natale, sur les berges du lac Léman. Au printemps 1783, âgé de dix-sept ans, poursuivi pour un vol commis chez un marchand d'Évian, Rosay fait un premier séjour de trois mois en prison à Thonon. En décembre 1785, voulant peut-être échapper à la marginalité, Rosay tente à nouveau l'expérience militaire. Il s'engage, en France, dans le régiment suisse de Courten. Sa nouvelle désertion est réprimée par la maréchaussée qui le conduit à nouveau en prison. Passé aux «verges », le jeune homme est expulsé du régiment avec le «congé jaune » qui le déclare indigne de servir le Roi. L'été suivant, rôdant dans la ville sarde de Carouge à la frontière de Genève, il s'enrôle dans le régiment suisse "Grison " au service de sa Majesté de Sardaigne. Après quelques semaines de service, il quitte clandestinement sa garnison et gagne certainement Genève. En effet, à l'époque de cette troisième désertion militaire, Rosay est arrêté une première fois à Genève sous le 
faux nom de Claude Gaillard. Poursuivi pour "effraction de pavillon", Rosay est condamné à être "censuré et mis hors de la ville par les chasses gueux $»^{8}$. Trois mois plus tard, sa « carrière criminelle " prend fin avec sa nouvelle arrestation qui fait suite à trois vols d'indiennes (tissus imprimés et fabriqués dans les manufactures de Genève). Pour Rosay, cette seconde incrimination est fatale : les auditeurs de justice vont percer sa véritable identité en reconstituant sa lourde biographie criminelle9. Trente-trois délits, dont plusieurs « vols » et « assassinats sur le grand chemin » : François Rosay, 22 ans, est un professionnel du crime ${ }^{10}$. Mesurant son « endurcissement dans le crime », le réquisitoire du procureur général François-André Naville requiert la peine capitale contre le brigand savoyard. Établissant la somme mathématique des crimes et délits dans le tableau des « charges et des délits " pour calculer positivement la peine requise selon le cumul des "circonstances aggravantes", le procureur général dresse un portrait à charge. Il retient notamment le cumul des crimes, la récidive, la préméditation, l'usage des armes, l'utilisation de nombreux faux noms et la multiplication des déguisements. Après délibération, les magistrats du Petit Conseil prononcent la peine capitale (pendaison) en suivant ainsi le réquisitoire du parquet. Par le biais d'un avocat désigné d'office, la demande en grâce que Rosay sollicite auprès du Conseil des CC (conseil supérieur entre le Conseil général des citoyens et le Petit Conseil de la République) échoue. Huit mois après son arrestation, sa sentence ayant été lue publiquement, Rosay est pendu à l'extérieur de la Porte neuve, face au couchant.

5 Reconstruisant à charge la biographie de Rosay, les sources judiciaires permettent d'affiner son profil physique. Les témoignages et le «Livre pour les signalements et les visites $»^{11}$ qui résume le signalement et la morphologie des prisonniers portraiturent Rosay. De petite taille, morphologie solide, Rosay est un beau jeune homme. Signes particuliers : yeux ambrés, joue droite marquée d'une cicatrice discrète. Son caractère trempé et sa parole menaçante s'accompagnent d'une force musculaire impressionnante. Les témoins le décrivent d'ailleurs comme « Hercule dont la massue faisait la terreur du Chablais $»^{12}$. Trois fois, il rompt les fers de la prison qu'un serrurier doit renforcer. Selon l'auditeur de justice qui l'interroge régulièrement, Rosay est continuellement remué par une " agitation naturelle». Le prisonnier évoque ses excès de violence, notamment lorsqu'il brise un violon sur la tête de son propriétaire parce que celui-ci arrête de jouer ${ }^{13}$. Claquemuré dans sa cellule, Rosay bricole habilement une clé pour ouvrir et fermer "à volonté " ses menottes. Considéré par les magistrats genevois comme un des pires brigands de son temps, il ne porte toutefois aucune marque corporelle d'infamie qui montre sa récidive. Avec son allure de soldat, de déserteur ou de recruteur, parfois riche et souvent pauvre, il erre sur les chemins, fréquente les cabarets dont il fait son foyer. Vagabond réclamant occasionnellement la charité, rétif au travail, Rosay vit de vols commis seul ou avec sa bande.

6 Cartouche, Mandrin : si la figure du bandit de grand chemin occupe dans l'imaginaire social une place notable, qu'en est-il de la réputation de François Rosay? Incriminé à Genève, il donne deux faux noms avant d'avouer sa véritable identité. Rosay n'est pas "signalé " dans la République: son arrestation est le fruit du hasard. Seule l'information judiciaire permet de reconstituer sa biographie, ses délits, soit sa «dangerosité ». À Genève, nulle réputation particulière-ni effroi social, ni glorification ambiguë dans une brochure d'échafaud - n'entoure le bandit savoyard. Son arrestation discrète tranche avec celle d'un autre bandit de grand chemin, Colomb de Battines de Coponay, noble savoyard déclassé, incriminé en 1777 pour meurtre. Lors 
de son transport à Genève, Colomb de Battines attire une foule de curieux ${ }^{14}$, alors que Rosay ne suscite que l'indifférence. Sauf une note du pasteur Picot qui le visite en prison selon l'usage des ministres « consolateurs », rien ne montre que Rosay intéresse le public hors des sphères judiciaires. Forgeant la crainte des communautés paysannes, sa mauvaise réputation n'a pas suinté hors du Chablais (Savoie) dont il est originaire. À Évian, Rosay appartient au «milieu » des individus suspects ou craints. Maints témoins (neuf sur onze) l'identifient sous son nom réel. Dans son village de Lugrin, tous reconnaissent que le «vaurien » et le « coquin » Rosay est un enfant du pays qui a mal tourné. Il rôde notamment la nuit pour «faire des mauvaises actions ». Certains paysans conviennent qu'ils se terrent chez eux une fois le soleil couché pour échapper à Rosay :

On n'osait pas aller par les chemins la nuit à cause de lui et on a été bien content d'apprendre qu'il était détenu ${ }^{15}$.

7 Hors de son « pays » qu'il terrorise - village, paroisse -, la crainte de Rosay s'atténue. Sa réputation d'homme endurci dans le crime est donc locale, même si durant son procès, le réquisitoire du procureur général en fait une figure majeure du banditisme. Si la culture juridique des magistrats genevois transforme Rosay en homo criminalis particulièrement dangereux, il reste un marginal dont la violence criminelle s'affirme au gré de ses vagabondages. Le milieu familial de Rosay montre son enracinement social dans la «vie fragile » de l'Ancien Régime. Né dans un foyer pauvre, quatrième de sept enfants, François Rosay rompt très vite les liens avec la domus familiale ${ }^{16}$. Selon quelques villageois de Lugrin, le père Rosay refuse de venir en aide à son fils incarcéré à Genève. Certains témoins l'ont simplement croisé, d'autres l'ont fréquenté, lui ont parlé, ont commercé ou bu avec lui. Avec son frère Joseph, François entretient une relation conflictuelle. Le litige fraternel porte notamment sur la question d'une solde datant de l'époque du régiment de Savoie. François réclame l'argent en prétendant que son frère, engagé avec lui, l'aurait empoché. Selon les cinquante-huit témoins qui déposent durant l'information ouverte contre Rosay, son milieu social est celui d'un paysan déclassé, fréquentant plus que de raison les cabarets, trop souvent sur le grand chemin. Détaché de sa famille, sans lien de travail, déserteur, sans le sou, Rosay est un marginal dans son groupe social. Pourtant, forgée par certains témoignages et son «testament de mort ", l'image du brigand vivant reclus dans les bois et ne sortant que la nuit pour rapiner les paysans, est réductrice. Rosay est bien inséré dans le réseau social de son temps. Il se déplace en Savoie (parfois en usant un faux nom pour échapper à son " signalement " judiciaire), commerce, fréquente les bateliers avec qui il traverse le lac Léman, offre à boire à maints compères de cabarets, loge dans les auberges $^{17}$.

Il est donc possible de corriger l'image stéréotypée du bandit-clandestinité, marginalité, endurcissement dans le crime - afin de lui donner sa dimension sociale et de mesurer que cette image s'enracine dans l'imaginaire de ceux que Rosay fréquente ou rapine. Notons tout d'abord que la procédure commence avec les dépositions et déclarations de neuf ouvriers de la fabrique d'indiennes des Eaux Vives, puisque l'arrestation de Rosay suit la plainte de son propriétaire. Les témoins qui fréquentent François Rosay sont des hommes et des femmes âgés de quinze à soixante ans. Douze ont une activité liée au cabaret et à la vente de boisson: cabaretiers, servantes de «bouchon » ou vendeur de vin et d'eau de vie en échoppe. Les « rixes » dont Rosay est accusé trouvent en ces lieux leurs motivations vindicatives, leurs enjeux sociaux et symboliques, notamment autour des tables de jeu. Sept autres témoins sont des ruraux : 
paysans, laboureurs ou " ouvrières de terre ", rencontrés sur le "grand chemin » : ils opposent souvent leur enracinement social à l'errance de Rosay. Sept encore sont marchands, trois sont bateliers et deux femmes lingères. D'autres témoins exercent des activités mal identifiables ou alors «libérales", à l'instar du "praticien notaire » de Lugrin, Pierre François. Parmi les victimes de Rosay, on remarque quatre paysans (ou journaliers), quatre cabaretiers, cinq marchands et deux soldats. En fait, le bandit Rosay est moins un marginal qu'un individu inséré dans un milieu social contemporain qui ne différencie pas nettement les « honnêtes gens » des « scélérats ".

Protagoniste majeur du banditisme associatif, le receleur confirme l'ancrage de Rosay dans les sociabilités de son temps et de sa région. Chargé d'écouler sur le marché le butin volé par les brigands, ayant un domicile fixe, le receleur est souvent marchand ou cabaretier ${ }^{18}$. Il achète à bas prix des biens, qu'il sait volés (voire qu'il a signalés aux voleurs) pour les revendre, moyennant un bénéfice. Aux franges de la criminalité organisée, le receleur est le personnage emblématique d'une société qui ne peut se distancier du crime. Ce lien économique illustre le rapport social implicite entre le voleur et l'acheteur final, lequel, sera peut-être un jour aussi dépouillé. Ainsi, sans passer par un intermédiaire, lorsque Rosay vend à Douvaine (village près de Genève), les indiennes volées à la fabrique des Eaux-Vives ${ }^{19}$, il est probable que l'acquéreur, pour parer à toute éventuelle accusation de complicité, se contente des explications évasives de Rosay sur leur prix et le fait que le tissu est encore mouillé. Si le vol et les atteintes à la propriété sont des crimes quotidiens durant l'Ancien Régime, l'attrait social pour le produit des larcins est important. Dans une société mal policée où les frontières entre les illégalismes et les normes sont poreuses, le goût du butin l'emporte sur la peur de la répression et permet de corriger la misère des milieux modestes. Le receleur tire profit de cet attrait pour le bénéfice du crime et joue ainsi le rôle d'intermédiaire (maillon social) entre le réseau des malfaiteurs et une certaine catégorie de consommateurs peu regardants sur l'origine des produits revendus.

\section{Association criminelle}

L'association criminelle résulte d'un regroupement de voleurs en une bande. Il s'agit de diviser le travail criminel tout en augmentant les chances de succès et en diminuant les risques répartis entre les membres de la bande ${ }^{20}$. Le «testament de mort» de Rosay confirme la structure du banditisme traditionnel. Repaire creusé dans les berges d'une rivière voisine de Genève (Hermance), indicateur qui repère les lieux à piller, guet qui couvre le travail de la bande ou détourne l'attention des victimes (Fornex «qui se fait un jeu du métier d'incendiaire $\aleph^{21}$ ), serrurier fabricant les fausses clefs, spécialiste du vol ou de l'écoulement du butin : la bande du chef Orléans que décrit Rosay montre la dimension sociale de la criminalité associative. Parfaitement organisée - Orléans tient à jour la liste des membres du réseau hiérarchiquement structuré - la solidarité n'appartient pas à la culture de la bande. Si les marchandises partagées comme provisions de bouche illustrent une forme de communautarisme social, les normes de l'association criminelle sont individualistes. Dans la bande d'Orléans, règnent la suspicion, les règlements de compte voire les assassinats (meurtre du bandit Musca dans le « testament de mort » de Rosay).

11 Au même titre que l'association criminelle, et liées à elle, l'armée et la prison sont deux autres étapes de la biographie de Rosay. Sa carrière de mercenaire compte trois 
engagements et autant de désertions. Replacé dans le contexte militaire de l'époque ${ }^{22}$, ce comportement est coutumier. Même si elle est sévèrement punie, la désertion est massive durant tout l'Ancien Régime. Si l'armée offre aux volontaires le cadre d'une institution d'intégration sociale et la sécurité d'une maigre solde, d'autres s'y retrouvent engagés contre leur gré. Emmenés par un « recruteur », ils ont signé sous la contrainte, l'emprise de l'alcool, voire alléchés par des promesses mensongères. Ces victimes sont souvent vagabonds, apprentis sans maître, repris de justice ou galériens libérés.

Quelles sont les conséquences des multiples enrôlements de Rosay? Premièrement, ses trois désertions successives accentuent sa dérive sociale en l'éloignant progressivement d'une « vie normale »- famille, travail, sociabilité rurale. Sa marginalité est accentuée par la « cartouche jaune » reçue après sa tentative de désertion de Courten ${ }^{23}$. Mendiant aux portes de Genève, Rosay exhibe ce congé infamant, lequel prouverait non seulement son incapacité à servir le Roi de France, mais également son impossibilité à se resocialiser. Chassé de l'armée, Rosay n'est-il pas ainsi acculé à la mendicité ? La marque d'infamie-plus morale que corporelle-grave sur Rosay le signe de sa marginalité. Pourtant, le régiment lui offrait l'opportunité d'échapper à sa «vie fragile ». Son premier engagement en Savoie représentait la " dernière tentative de sa famille à le ranger à son devoir $\aleph^{24}$. L'armée peut être un emploi comme un autre et, par-là, assurer la stabilité financière et sociale du soldat, selon l'exemple de Joseph, l'aîné de François resté au régiment de Savoie. Dans la troupe, le contact avec des pairs peu fréquentables ne semble pas réellement influencer Rosay. Au contraire, c'est lui qui joue avec le destin de quelques-uns de ses compagnons d'armes - notamment Duret qu'il pousse à déserter ${ }^{25}$. Finalement, son passage dans les divers régiments ne lui a pas offert l'opportunité de s'exercer au maniement des armes, car il n'y est jamais resté suffisamment longtemps pour avoir eu l'occasion d'y toucher ${ }^{26}$. S'il est certain que la biographie criminelle de Rosay recoupe son expérience militaire, il est moins certain que celle-ci ait réellement marqué sa destinée, contrairement à son expérience carcérale.

Rosay fait cinq séjours en prison ${ }^{27}$. Il subit un premier contact avec les fers à Évian durée indéterminée - et reste enfermé trois mois à Thonon suite à divers larcins. À Limoges, il passe quelques jours dans les geôles de l'armée française suite à sa tentative de désertion. À cela s'ajoute un premier séjour de 4 jours de prison à Genève (été 1786), ayant été saisi pour une " effraction de pavillon ». Le 7 novembre, il est arrêté pour la seconde fois à Genève. Il ne sort de son cachot que pour monter sur le gibet. Relaté dans son « testament de mort ", l'épisode carcéral de Thonon, qui illustre la complicité entre le geôlier délinquant et les prisonniers chargés par lui de campagnes de vols nocturnes, est fondateur de la biographie criminelle de Rosay ${ }^{28}$. Espace avant tout correctif ou de sécurité avant le procès ${ }^{29}$, la prison d'Ancien Régime livre ici sa véritable dimension de fabrique d'homo criminalis. La promiscuité entre condamnés récidivistes et jeunes délinquants est propice à la reproduction du crime, voire à son organisation en association. Comme le montre le témoignage de Rosay, la prison favorise alors le renforcement social des bandes. Abusant de sa position d'autorité sur les prévenus et voulant améliorer son modeste revenu, le geôlier de la prison de Thonon planifie une forme de criminalité associative en les envoyant piller les maisons des environs. Chaque détenu remplit un rôle bien précis dans cette forme de crime organisé depuis la prison; ainsi, Rosay est chargé d'écouler le butin hors de la prison, car il n'est pas connu 
à Thonon. Il convient dans son "testament de mort", que sa "vie dissolue " de prisonnier a contribué à le « corromp[re] davantage».

\section{Mourir sur le gibet}

14 Un jour après que le Conseil des CC ait refusé son recours en grâce, Rosay sort de sa geôle pour marcher vers le gibet, dressé en dehors de la ville à la porte Neuve. Son exécution est conforme au rite pénal de l'Ancien Régime. Elle vise notamment à intimider le public et à montrer la mort du larron repenti ${ }^{30}$. L'exécution est précédée par la rédaction du «testament de mort». Celui de Rosay contient les aveux faits en prison avant et après le jugement, ainsi que ceux prononcés sur l'échafaud. Les déclarations sont réécrites par les magistrats («verbal »). Celui-ci est relu une dernière fois au condamné qui demande à corriger certains passages - maints passages tracés et marginalia. Contrairement à d'autres juridictions où la pratique judiciaire utilise souvent ce rituel d'échafaud, à Genève, la faible dimension de la peine capitale (notamment dans la seconde moitié du XVIII siècle) raréfie les «testaments de mort ». Comment expliquer l'importance de celui de Rosay?

En cas de condamnation à mort, l'accompagnement des condamnés par les "pasteurs consolateurs " est coutumier à Genève ${ }^{31}$. Deux pasteurs doivent préparer le "patient " et le « réconforter ». Si les ministres de Dieu s'engagent à " consoler » le condamné, ils œuvrent aussi dans la logique pénale : les aveux arrachés aux condamnés serviront à la «satisfaction des juges pour lesquels la plus légère apparence de doute est un tourment $\aleph^{32}$. La présence de deux ministres près du condamné mené vers l'échafaud joue sur deux registres. Sur le plan spirituel, le salut de «l'infortuné » repose sur la repentance. Celle-ci commence avec la confession du crime commis. À Genève, comme ailleurs en Europe continentale, l'aveu appartient au régime probatoire de la procédure inquisitoire ${ }^{33}$. En outre, le «testament de mort» confirme et précise toutes les « circonstances » morales et matérielles du délit jugé, tout en permettant au patient de dénoncer le cas échéant des complicités tues durant l'instruction judiciaire. Rosay, face à la corde qui l'attend, dénonce une vingtaine de complices, signale pour la première fois l'existence du repaire proche de Genève, puis détaille certains épisodes de sa biographie d'homme coupable.

16 La présence, au tribunal et sur l'échafaud, du père Édouard, un capucin de Gex, irrite fortement les pasteurs genevois et "le public [le voit] avec beaucoup de peine $»^{34}$. Dans la Genève protestante, où le sacrement confessionnel n'existe pas, le ministère catholique demandé par Rosay (catholique) illustre l'importance attachée aux ultimes aveux. La «confession» de Rosay aboutit à un long récit quasi autobiographique. Par ailleurs, l'étendue du procès, la longueur de l'instruction et les implications sociales de l'association criminelle soulignées plusieurs fois par le Petit Conseil de la République (maintes notations dans le registre du Conseil), expliquent certainement la minutie mise à recueillir les ultimes aveux de Rosay, perçu peut-être comme un gros poisson du banditisme régional ${ }^{35}$. Dans la logique répressive, en dramatisant le cas de François Rosay, le "testament de mort » sert, in fine, à produire la vérité à charge sur la dangerosité de la criminalité associative.

17 L'importance des déclarations contenues dans le «testament de mort » lui donne une portée juridique considérable. Dès sa réception, les autorités lancent des recherches, certes infructueuses, pour trouver le repaire et d'éventuels complices. Le «testament 
de mort» structure la coopération judiciaire avec d'autres juridictions-Savoie, Lausanne, montagnes neuchâteloises - pour donner suite aux déclarations de Rosay. Les magistrats peinent à retrouver les complices dénoncés, ainsi que les vestiges des vols confessés. Lorsqu'ils se renseignent chez leurs confrères, rien ne semble confirmer les aveux de Rosay, ce qui nous impose une certaine prudence quant à la vérité de ses déclarations. Le "testament de mort» dramatise le crime et sa réparation pénale. Il illustre le décalage qui existe entre François Rosay, né dans une famille misérable, jeune homme agité, endurci dans le crime par son expérience carcérale, auteur d'un seul meurtre - trois autres "assassinats» dont il est inculpé étant en fait des agressions - et l'imaginaire du banditisme. À usage interne aux sphères judiciaires, le «testament de mort » est un élément constitutif de la notion de dangerosité criminelle à l'époque moderne. Malgré le réquisitoire capital du procureur général, pour qui chaque forfait pris séparément doit être « remis à une foule d'autres délits commis par un homme notoirement connu pour ses crimes [et] doit concourir avec tous les autres pour nécessiter [les juges] à prononcer la peine de mort $»^{36}$, la figure du brigand devient un peu dérisoire à la relecture du dossier. "Hercule» du crime, François Rosay, vagabond au couteau facile, laisse toutefois un récit singulier et fascinant. Cette autobiographie induite par la justice illustre, une fois encore, la richesse et les enjeux épistémologiques des archives judiciaires pour l'histoire sociale des justiciables à l'époque moderne.

\section{Sources manuscrites}

\section{Archives d'État de Genève}

AEG PC (I) 14922 [Procès Claude Gaillard alias François Rosay].

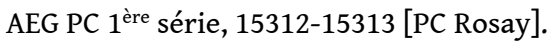

AEG PC $1^{\text {ère }}$ série $15030,15303,15305,15159$.

AEG Jur. Pen. $\mathrm{H} 2 \mathrm{n}^{\circ} 2$ « Registre de signalements et de visites faites à Genève de condamnés, de prisonniers et de suspects (1775-1793)».

AEG Archives de famille, Naville, XIV.A - 6/2 « Notes extraites d'un livre de notes de Mr P. Picot, pasteur et professeur ».

AEG Cp. Past. R34, « Registre de la vénérable compagnie des pasteurs », p. 50.

AEG RC 274, « Registre du Conseil » 1773, fol. 702, « Mémoire des pasteurs consolateurs » du 20 décembre 1773.

AEG RC 290-291, « Registre du Conseil » (1786-1787). 


\section{Archives départementales de Haute-Savoie}

ADHS 5Mi 932 n $^{\circ}$ « Registre des baptêmes, 1739-751 » et $n^{\circ} 11$ « Registre des baptêmes, 1751-1793».

\section{Sources imprimées}

Ferrière C.-J. de; Nouvelle introduction à la pratique : contenant l'explication des termes de pratique, de droit et de coutume, avec les juridictions de France, Bruxelles, 1739.

Précis historique de la vie de Coponex [Copponnais, Copponex], Lyon, 1764.

\section{Références}

Bée M., Le spectacle de l'exécution dans la France de l'Ancien Régime, Annales ESC, 1983, 4, pp. 843-862.

Briegel F., La clémence du glaive : plaider pour les criminels au siècle des Lumières à Genève, Crime, Histoire et Société, 2000, 4, 1, pp. 9-29.

Carbasse J.-M., Histoire du droit pénal et de la justice criminelle, Paris, PUF, 2000.

Chagniot J., Paris et l'armée au XVIII siècle : étude politique et sociale, Paris, Economica, 1985.

Corvisier A. (dir.), Histoire militaire de la France, Paris, PUF, 1992.

Crépin M.-Y., Le chant du cygne du condamné : les testaments de mort en Bretagne au XVIII ${ }^{\mathrm{e}}$ siècle, Revue histoire et droit, 1992, 4, pp. 491-509.

Deyon P., Le temps des prisons : Essai sur l'histoire de la délinquance et les origines du système pénitentiaire, Paris, Éditions universitaires, 1975.

Dubied C., « La lie de la canaille » : larrons, brigands et filous de profession, la répression du banditisme à Genève (1682-1792), Crime, Histoire et Société, 2001, 5, 2, pp. 107-132.

Egmond F., Underworlds : Organized Crime in the Netherlands 1650-1800, Cambridge, Polity Press, 1993.

Foucault M., Surveiller et punir : naissance de la prison, Paris, Gallimard, 1975.

Langlois M., Lanhers Y., Confessions et jugements de criminels au Parlement de Paris, Paris, SEVPEN, 1971.

Porret M., Mourir sur l'échafaud à Genève au XVIII siècle, Déviance et société, 1991, 15, 4, pp. 381-405.

Porret M., Le crime et ses circonstances : de l'esprit de l'arbitraire au siècle des Lumières selon les réquisitoires des procureurs généraux de Genève, Genève, Droz, 1995 (a).

Porret M., La biographie des scélérats ou les circonstances de la dangerosité criminelle durant l'Ancien Régime, Traverses : biographie, 1995 (b), 2, pp. 55-64.

Sharpe A. J., « Last dying speeches » : Religion, ideology and public execution in seventeenthcentury England, Past and Present : Journal of historical studies, 1985, 107, pp. 144-167. 


\section{ANNEXES}

\section{Testament de mort de François Rosay ${ }^{37}$}

\section{Avertissement}

Les numéros entre crochets indiquent les pages du texte original. Les passages soulignés dans l'original sont en italique dans notre transcription. Nous avons utilisé l'orthographe moderne et adapté la ponctuation afin de rendre la lecture du texte plus aisée. Les guillemets qui signalent les citations de Rosay (discours direct) ont été rajoutés par nous. Certains noms propres apparaissant orthographiés de manière aléatoire, un choix arbitraire a été fait afin d'utiliser une seule orthographe par nom pour toute la transcription. Les noms de lieux sont écrits dans leur orthographe moderne et dans les cas problématiques, le nom figurant dans le texte est précisé en note. Les mots et noms propres incertains sont signalés par les crochets []. De plus, certains paragraphes comprennent des rajouts inscrits dans la marge ou des suppressions qui sont probablement des corrections proposées par Rosay suite à la relecture du testament. Il a été tenu compte de ces corrections sans qu'elles soient à chaque fois signalées.

[1] Du 14 juin 1787

Aveux faits par François Rosay, de Lugrin en Chablais, après la clôture de la procédure instruite sur ses délits et confirmés par lui le jour de son supplice et peu de moments avant sa mort.

Il a dit que, depuis longtemps, il faisait partie d'une bande de voleurs, à la tête de laquelle est un nommé Orléans, homme grand d'environ 5 pieds 10 pouces $^{38}$, de mauvaise mine, reconnaissable par sa tête trop petite pour son corps. Ils l'appelaient le Capitaine. Que cet Orléans s'est creusé un repaire, soit cave, dans les [crâtes] ${ }^{39}$ de la rivière dite l'Hermance, située entre les Bois de Jussy et le Coteau de Boisy. Que lui, Rosay, était arrivé dans ce repaire pour la première fois avec Orléans, qu'il avait trouvé près de Genève et $\mathrm{y}$ avait été conduit par Salins peu de temps après être revenu des troupes du Piémont ${ }^{40}$ et avant même d'aller servir en Courten ${ }^{41}$. Juilard, avec qui il était parti, l'ayant engagé à quitter le repaire, le dit Juilard est, dit-il, resté dans Courten.

Rosay a ajouté que le repaire est grand au point qu'ils s'y sont trouvés une fois 18 ensemble, mais que plusieurs sont morts depuis, deux ayant été arrêtés à [Jogne $]^{42}$ en Franche-Comté, au moment qu'ils volaient dans l'auberge de cette ville qui a pour enseigne la Couronne, que c'était l'année dernière et qu'ils n'ont pas [2] reparu dès lors. Que le nommé Salins fut tué peu après à St-Paul, au dessus d'Évian, d'un coup de faux que lui donna un paysan dans la maison duquel il cherchait à voler. Rosay convient qu'il était de ce vol, mais qu'il se sauva. Il ajoute que lorsqu'il a été arrêté, il ne restait plus que 13 habitués du repaire, [à] savoir 11 hommes et 2 femmes. Le chemin que Rosay indique est d'aller à [Meinier] ${ }^{43}$, puis de prendre la direction du Coteau de Boisy en tirant un peu sur la gauche, tout à travers champs, jusqu'à ce que l'on soit à l'Hermance. Il assure qu'en le suivant, on ne peut manquer de trouver l'endroit où est situé le dit repaire, lequel endroit est reconnaissable tant à une petite mare qu'ils ont creusée aux environs pour avoir de l'eau quand le ruisseau est à sec, qu'à une grosse 
pierre qui est voisine de l'entrée et qui, à moitié hors de terre, porte des traces de feu, parce qu'on a, pense-t-il, enfumé quelque renard terré sous elle.

Il ajoute que cette entrée, qui ressemble à un trou de blaireau est cachée par un gros buisson de chêne, observant même qu'il faut détourner une partie des branches de ce buisson pour l'apercevoir. Que cette entrée est basse et fort étroite, que l'on ne peut pénétrer dans l'intérieur qu'en se blottissant contre terre et entrant à genoux. Mais qu'après avoir fait ainsi deux pas, le repaire s'agrandit, et qu'on peut y être debout, [3] qu'il est long d'environ 8 pieds, et large de plus de $5^{44}$. Qu'ils en ont soutenu la voûte par des plateaux de bois, étayés avec des pieux, qu'ils l'ont carrelée avec des tuiles qu'ils avaient volées à la fabrique de Crévy. Qu'ils y ont pratiqué une espèce de lit de camp, dont l'intérieur est disposé pour contenir les vols et qu'ils étendent de la paille sur la partie extérieure qui leur sert de lit. Qu'ils tiennent leur argent dans un pot de terre qui est enfoncé dans le terrain et couvert de fer blanc. Que ce pot est placé sous le lit de camp et caché par des tuiles, et même par de la terre. Qu'ils ont un fourneau de fer et que c'est là que les femmes font la cuisine, mais qu'ils ne l'allument que la nuit, dans la crainte que la fumée ne les décèle, attendu que le tuyau sort du repaire tout contre terre. Il dit que la paille et le fourneau ont été achetés chez Chapelle ${ }^{45}$, aubergiste à Corsier, ainsi qu'une barrique de vin qu'ils y portèrent. Il ajoute qu'ensuite ils firent des paillasses avec des draps volés et qu'ils les placèrent sur le lit de camp pour y être plus commodément.

Pressé de décrire plus précisément la place où ce repaire est situé, Rosay a assuré ne pouvoir le faire d'une manière plus exacte, mais qu'on le reconnaîtra parce que, de la hauteur qui domine le repaire, on voit un village qui n'en est pas fort éloigné et qu'il croit être [Les Étolles] ${ }^{46}$, [4] distantes d'environ une demi-lieue du repaire ${ }^{47}$.

D'ordinaire, a dit Rosay, les voleurs se tiennent là pendant le jour et ils se répandent le soir dans la campagne, les uns d'un côté, les autres de l'autre, pour chercher des coups à faire. Il dit que lui, Rosay, les conduisait au nombre de huit dans la montagne qui est au-dessus de Thonon, qu'ils y volèrent 15 fromages; qu'une partie fut portée au repaire pour leur servir de provisions et qu'ils vendirent le reste. Que Chapelle en acheta pour 20 livres, qu'il paya sur le pied ${ }^{48}$ de $7 \frac{1}{2}$ [sous] de Genève la livre. Les voleurs du repaire font, dit-il, leur provision de pain le samedi au soir et pour l'ordinaire à Chêne chez $\operatorname{mad}[\mathrm{emoiselle]} \mathrm{[Jolit]} \mathrm{ou} \mathrm{quelquefois} \mathrm{le} \mathrm{dimanche} \mathrm{matin} \mathrm{ou} \mathrm{à} \mathrm{Vésenaz.} \mathrm{En} \mathrm{général,} \mathrm{ils} \mathrm{ne}$ sortent jamais du repaire que la nuit et ils y rentrent avant le jour. Les deux femmes sont communes à la troupe. On vole souvent des poules, mais à un certain éloignement pour ne pas occasionner des recherches et le repaire est plein de plumes. C'est surtout vers Noël qu'on va faire des coups et du temps des messes de minuit. Ils avaient, ajoute Rosay, un petit livre sur lequel ils inscrivaient les noms de ceux qui formaient ou qui se joignaient à cette bande. Il a ajouté encore, qu'emprisonné ici depuis sept mois, il ne sait si tout cela n'a pas changé depuis lors. Mais il assure que c'était au dit repaire où ils allaient se rendre, Fornex et lui, lorsqu'ils furent arrêtés et conduits dans les prisons de cette ville. Ils avaient, dit-il, des armes dans ce repaire : un fusil à deux coups, volé par Orléans chez Mons[ieur] l'avocat de lois au-dessus de Thonon; des pistolets et les munitions nécessaires; des sabres et une épée que [5] lui Rosay acheta des domestiques de Serre ${ }^{49}$ de Pressy, en juillet dernier, pour un écu de six livres et qu'il y a laissé le tout pour se défendre, assure-t-il, s'ils étaient attaqués. Ceux qu'il y a vus et avec lesquels il a été en relation sont : 
Orléans.

Susdit, taille de 5 pieds 11 pouces ${ }^{50}$, ut supra. C'est lui, dit Rosay, qui va avec les deux femmes, faire la provision du pain à Chêne. C'est aussi lui qui, avec Lorrain la Toccante, Musca $^{51}$ et Bougeon ${ }^{52}$, ont fait le vol du curé de Thônex, à qui ils prirent l'été dernier son argent consistant en 15 à 1600 livres $^{53}$. Rosay dit qu'alors il était chez les Gru. Suivant lui Orléans est faux-monnayeur, métier qu'il entend bien, ayant travaillé dans les ateliers de l'hôtel des monnaies à Paris. Il a essayé, l'année dernière, de faire des écus neufs avec un moule de plomb. Mais le moule étant informe et la chose n'ayant pu réussir, il travaillait, dit-il, à en faire un autre en métal mêlé avec de l'étain fin qu'il avait acheté à Genève, lorsque lui Rosay quitta le repaire, c'est-à-dire peu de jours avant l'assassinat de Musca. Cet Orléans, ajoute Rosay, c'est aussi un grand faiseur de fausses clefs. Il a toujours avec lui, dit Rosay, des montants de clefs vides ${ }^{54}$ et des pannetons ${ }^{55}$ prêts à y être placés, selon les serrures. Il a ajouté enfin qu'Orléans cherchait à grossir sa bande, de manière à avoir 25 hommes sûrs, pour essayer de voler le couvent de Ripaille, disant qu'après ce coup il n'aurait qu'à se [6] se reposer, qu'il serait riche.

\section{Lorrain dit la Toccante ${ }^{56}$.}

De Toulouse. C'est lui qui fit le vol chez les dem[oise]lles Tremblay au Carre. Rosay assure qu'il fut commis, sur l'indication de Bougeon du Carre, par ledit Bougeon, par Bougeon de Maraîche, Orléans, Lorrain susdit, Fornex et Bruchon et que tous les effets avaient été portés dans le repaire. Les Ruche de Choulex avaient été soupçonnés d'être les auteurs de ce vol. Une partie de ces effets ont été ensuite vendus à Chapelle de Corsier et une autre partie à la sœur de la femme Simon, à Chêne sur Genève. Rosay a parlé de Lorrain comme d'un homme aussi adroit que déterminé, qui se fait un jeu des plus grands crimes, comme des plus difficiles et qui depuis qu'il court la campagne en a commis de tous genres. On verra, dans la suite de ces confessions de Rosay, les crimes auxquels il l'accuse d'avoir participé. Lorrain vient, dit-il, assez souvent dans cette ville, mais il couche à Carouge. Quand il a des effets à vendre, il les remet à une gagère qui demeure au haut de Coutance, tout près du Mouton. C'est une petite femme, vieille et maigre (ce doit être la nommée Faure) ${ }^{57}$. Il prétend qu'elle achète tout, quoiqu'elle sache bien que [cela] a été volé. Lorrain est aussi en relation avec la jeune de Du Fornay ${ }^{58}$, sœur de celui qui mendie, et a été mis deux fois hors de cette ville; quoiqu'elle soit domiciliée à Carouge [7] elle ne laisse pas d'être chaque jour ici, où elle fait le métier de revendeuse. Elle se tient aux rues basses du Lion d'Or ou entre les deux ponts. Lorrain lui remet son argent et ses effets les plus précieux.

$$
\text { Joseph Fornex }{ }^{59} \text {. }
$$

Avec qui Rosay fut arrêté et emprisonné, qu'on relâcha le croyant innocent et qu'il dit être le plus déterminé scélérat ayant commis des crimes de toutes espèces, et étant ce qu'ils appellent à tout. Rosay a raconté, comme un tour de gentillesse, que se trouvant près de la Tourronde en Chablais, avec le susdit Fornex et un nommé Jacquier de Lugrin (qui est aux galères pour un vol de cierges dans une église de Lugrin) n'ayant ni argent ni provisions et ne sachant où en prendre, parce que connu dans les environs pour gens dangereux, tout le monde les fuyait, Fornex s'avisa de mettre le feu à un grand tas de bois, ce qui attira tout le voisinage, tant par l'éclat de l'incendie que pour l'arrêter. Pendant ce temps, ils se glissèrent dans les maisons voisines et s'y chargèrent de pains, de fromages et même de miel. Rosay ajoute, en riant, que Fornex ne sachant dans quoi emporter ce miel, s'avisa de creuser un pain, de l'en remplir et de le charger sur sa tête. 
Mais que le miel ayant bientôt coulé, il en eut le visage et l'habit couverts, ce qui les amusa beaucoup. L'on a vu, ci-devant, que Fornex était l'un des [8] auteurs du vol fait au Carre, chez les Demois[e]lles Tremblay. Rosay prétend se souvenir avoir entendu le dit Fornex proposer à Orléans un vol au village de Marin en Chablais, ce qu'Orléans refusa, parce que pour y réussir, il fallait mettre le feu à une maison. Fornex, dit-il, se fait un jeu du métier d'incendiaire.

$$
\text { Du Fornay }{ }^{60} \text {. }
$$

De Carouge. On verra, ci-après, qu'il fut le complice de Rosay et de Lorrain, dans le vol fait à Vevey le 28 juillet dernier ${ }^{61}$, dans celui fait à Pierre Levray à Lugrin, dans celui fait à un contrebandier à Chêne [sur] Genève et

\section{Claude Bruchon.}

De Fribourg, âgé de 25 ans, taille de 5 pieds 2 pouces $^{62}$, nez rouge. L'on a vu qu'il était complice du vol fait chez les D[emoise]lles Tremblay.

$$
\text { Cadet. }
$$

Né à Montpellier, âgé d'environ 26 ans.

$$
\text { Pierre Bougeon de Maraîche. }
$$

Brigand qui a été pendu en effigie ${ }^{63}$ à Thonon. C'est lui qui est accusé d'avoir commis l'assassinat dont Rosay parla à Vevey devant des bateliers qui ont déposé à la procédure, mais qu'il a désavoué à sa mort.

\section{[9]Bougeon.}

Du Carre, indicateur et l'un des voleurs de la campagne des D[emoise]lles Tremblay.

Antoine Saviot.

De Novara en Piémont, taille de 5 pieds 4 pouces $^{64}$, gravé de petite vérole, âgé de 30 ans.

$$
\text { Jacques Crique. }
$$

François, âgé de 18 ans, taille de 5 pieds 2 pouces $^{65}$.

$$
\text { Claudy Ducas. }
$$

De Contre-Fontaine, taille de 5 pieds, 3 pouces $^{66}$, âgé de 36 ans.

$$
\text { Jacques Barladin. }
$$

Espagnol, âgé de 28 ans, taille de 5 pieds, 3 pouces, verrue comme un pois à la joue gauche, petit visage maigre, à tout.

$$
\text { Jean le Blondin. }
$$

Celui dont il est parlé dans la procédure de Bassière, dit Courte-botte ${ }^{67}$, désigné comme [un de] ces fameux faiseurs de fausses clefs et qui pourrait bien être le camarade de Blanchard, qui lui écrivait des galères de Brest et lui marquait que, son temps devant bientôt finir, il irait bientôt le rejoindre à Moillesulaz près de Chêne.

$$
\text { Joseph Antogniate }{ }^{68} \text {. }
$$

Piémontais, prévenu d'être le complice de Rosay dans le meurtre de Musca. [Il n'habite dans] le repaire que depuis environ un an. Complice de Rosay pour le meurtre de Musca, les vols d'indiennes et autres ${ }^{69}$, le tout détaillé ci-après. 
[10]Claude Salins qui fut tué à [Vinzier] ${ }^{70}$ au-dessus de St-Paul. Il nomme aussi François Jaquier, et Morel qui ont disparu depuis [Jogne]. Marie Grachsel de Berne, qui a été fouettée et chassée de cette ville, pour libertinage et la nommée Claudinette, de Lons-leSaunier.

Rosay, continuant ses confessions, a dit qu'il fit la connaissance de Lorrain la Toccante lorsque celui-ci sortait des prisons de Notre-Dame-des-Hermites ${ }^{71}$, ainsi que Wagnat de Thonon, son associé, après y avoir été l'un et l'autre fouettés et marqués pour une tentative de vol faite sur les vases de l'Église ${ }^{72}$. Ce Wagnat est celui qui apporte en cette ville les lettres de Thonon, quand le messager ne vient pas.

Que peu après cette connaissance, ils furent dans le Comté de Neuchâtel, et qu'en octobre 1785 (ce devait être le 28) ou au Locle ou à La Chaux-de-Fonds, ils volèrent chez Mr Rillaud une assez grande quantité de vaisselle d'argent ${ }^{73}$. Qu'ils passèrent par une fenêtre qui est près d'un four et qui a vue sur un jardin. [11] Qu'en y allant, ils s'arrêtèrent chez un homme et une femme qui vendent de la [mercerie et des liqueurs] et qui habitent le lieu-dit Le Chalet-à-Gobet, dans le Jura, à une lieue en-delà de Lausanne, sur la route de Moudon, à gauche en allant, et sur une petite élévation. Ils leurs dirent : «Préparez des louis, nous vous apporterons des effets » et que la femme répondit: «Allez seulement, les louis ne vous manqueront pas ». Il désigne le mari comme étant petit, brun, fort gravé de petite vérole, et sous le nom de Jean-François. La femme dit-il, est petite, maigre. Rosay et Lorrain l'appelaient la Richette. Elle tenait, dit Rosay, toujours bien des écus neufs, dans un sac de peau qu'elle avait dans sa garde-robe. Elle donna du coup trente louis à Lorrain, et à Claude Salins pour ce qu'ils lui apportèrent. "Ces gens, dit Rosay, reçoivent les voleurs " ${ }^{74}$. Lorrain est avec eux en grande relation. Rosay qui avait été chargé du paquet, y arriva si fatigué qu'il lui fallut, dit-il, plusieurs jours, et une grande quantité d'eau de vie et de lait pour se remettre. Salins, celui qui a été tué à St-Paul, était pour lors avec eux. Ils sont tellement attachés aux voleurs qui leur vendent ce qu'ils prennent, que Rosay disait, « si je sortais d'ici demain sans un sol, et que je fusse chez eux, ils m'en fourniraient pour me remonter ». Ces gens achetèrent la vaisselle susdite, il y en avait pour plus de 80 louis. Rosay ajoute qu'il escamota, sur le total, trois couteaux d'argent, [12] qu'il a apportés ici, dès lors, à son frère Gallay qui fit mine de les retenir pendant quelques mois, et enfin lui paya sur le pied d'un écu la pièce. Après le vol de vaisselle, ils prirent la route de France. Lorrain, Salins et lui, traversèrent le Doubs et vinrent rentrer en Suisse par La Sarraz. Rosay, après avoir donné ces détails, les as retranchés en partie le jour de sa mort, prétendant n'avoir pas participé à ce vol de vaisselle, l'avait seulement apporté de Lorrain et Salins qui l'avaient exécuté et leur avait escamoté ${ }^{75}$ les trois couteaux sur le dit vol.

Rosay demanda si, depuis son emprisonnement, il ne s'était pas commis un vol considérable à Vevey. Interrogé sur la raison qui lui faisait faire cette demande, il a répondu que c'est parce que Lorrain avait minuté d'enlever tout l'argent du Sieur Collomb $^{76}$, marchand de fer et agioteur ${ }^{77}$ au dit Vevey, qu'ils avaient été ensemble chez lui pour examiner le local, et que d'après cela, Lorrain avait résolu de creuser pardessous la porte d'entrée pour parvenir dans le comptoir, et qu'avec un ciseau il devait faire sauter la porte de l'armoire servant de coffre fort, laquelle est en fer et pratiquée dans le mur.

Il a ajouté que rien n'était plus vrai que la déposition qu'avaient fait deux jeunes bateliers d'Évian, qui disaient l'avoir vu au Pont d'Allaman, ayant une grande quantité 
d'écus de six livres. Que c'était le produit d'un vol qu'ils venaient de faire à Vevey, Lorrain et lui, qu'il avait partagé avec Lorrain. Qu'il avait trouvé son Lorrain chez le cordonnier au Petit-Saconnex vers le 23 juillet dernier (1786) et lorsqu'il sortait avec Du Fornay des prisons $\mathrm{d}^{\prime} \mathrm{ici}^{78}$, que Lorrain lui proposa d'aller en Suisse avec lui, ce qu'il fit. Et qu'étant à Vevey le 28 dudit mois de juillet, ils avaient ouvert le mur d'un comptoir, y étaient entrés, en avaient emporté 104 écus neufs qu'ils avaient partagés avec Lorrain, son complice, et que c'était ces écus qu'il avait dans son mouchoir lorsqu'il vit ces jeunes gens, et qu'il les fit bruiner à dessein et pour leur faire bien voir qu'il n'était pas dans la misère.

[13] Il a raconté que le nommé Moille de Lugrin et lui avaient volé à la foire de Crête, sur Thonon, une caisse remplie de culottes de peau, qu'elles appartenaient à un marchand suisse, qu'ils la portèrent chez un homme d'Évian, nommé Guerin d'Hauteville, qui était ci-devant cordonnier, mais qui par le profit qu'il a fait avec les voleurs, a été en état de lever une grande boutique de fer, qu'il leur donna pour la caisse de culottes, trois louis à chacun. Il a ajouté qu'ayant volé, à la foire de Thonon, une quantité de mouchoirs de soie, ce Guerin les lui acheta et ne voulut les lui payer que deux livres la pièce. Ayant fait un semblable coup à une autre foire, il fut arrêté et conduit aux prisons de Thonon, où il dénonça Guerin comme ayant acheté son vol, qu'on le força juridiquement à le rendre, mais qu'il ne fut pas puni.

Il a dit, de plus, qu'il était bien étonné que l'aubergiste Chapelle fut venu déposer contre lui, qu'il n'était rien moins que scrupuleux, puisque Jean le Blondin, Fornex et Bruchon ayant volé, dans la Montagne d'Abondance, deux juments et les ayant amenées chez lui, il se chargea de les aller vendre. Qu'il leur dit les avoir vendues à la foire de Gaillard, et les avoir données pour 8 louis qu'il leur remet, quoiqu'ils aient su depuis qu'il en avait reçu 13 en payement.

[14] Lorsqu'on lui a parlé du meurtre d'un marchand que lui, Rosay, et Rougeon sont accusés d'avoir tué, il se mit a rire et finit par répondre : «je n'ai pas assassiné, mais il faut bien consentir quelquefois à ce qu'on ne peut pas empêcher ». Il ne disconvint pas précisément d'avoir eu connaissance de ce crime et part à son produit, mais le matin du jour de son exécution, Rosay a nié d'avoir aucune connaissance de cet assassinat.

Questionné sur ce qui l'avait jeté si avant dans le crime, il a répondu que la débauche lui fit commettre les premiers, et que ce qui l'a achevé, c'est son séjour dans les prisons de Thonon. Il dit qu'y ayant été renfermé en mai 1783 pour avoir volé des mouchoirs et des boucles, à la sollicitation de Guerin d'Hauteville, il y trouva les nommés Duvillard de Chesnes, Michel Morassel de Lilly près de Bons et un Allemand, tous grands voleurs qui, liés avec le geôlier des dites prisons et voleur lui-même, l'associèrent aux coups qu'ils faisaient. Il assure que ce geôlier les faisait sortir chaque jour et surtout avec eux, que tous ensemble ils se répandaient dans la campagne, y volaient ce qui pouvait être à leur portée et rentrant aux prisons avant le jour, en faisaient le réceptacle des effets qu'ils avaient pris.

[15] Ce geôlier se nomme, dit-il, Benoît, sa femme Marguerite et ils avaient un fils dont le nom est Nicolas. On leur a ôté la garde des prisons depuis quelque temps et chassé le père de Thonon.

Rosay raconte qu'ils ne sortaient jamais de la prison que chargés de sacs, de haches et autres outils propres à faire sauter les portes. Que leur premier vol fut au nommé Cacha Martine, marchand à Évian, qu'ils forcèrent sa boutique, prirent tout ce qu'elle 
contenait et le portèrent aux prisons. Le second vol fut chez un pêcheur de Meillerie à qui il prirent un brochet de 20 livres, des carpes et des féras. Qu'il s'en souvient d'autant mieux que le geôlier fut mordu à la main par le brochet et que lui, Rosay, fut vendre ce poisson aux Minimes de Thonon qui le lui payèrent 12 livres du Piémont. Que le geôlier lui ouvrit les prisons, quoique en plein jour pour qu'il [vendit] ce poisson, parce que n'ayant pas encore été entendu, les juges ne le connaissaient pas. Le troisième vol se commit à St-Paul sur Évian où, ayant forcé un grenier, ils prirent 5 coupes de blé et des chemises. Le $4^{\mathrm{e}}$ se fit à [Liélui] ${ }^{79}$ dans la montagne. Ils volèrent des fromages et du beurre. Le $5^{\mathrm{e}}$ à [Armoins] ${ }^{80}$ sur Thonon, ils s'emparèrent d'une quantité de bled et de deux barriques d'eau de cerises qui étaient préparées pour être transportées en Suisse. Le $6^{\mathrm{e}}$ à Thonon même, ils volèrent chez le sieur De la Chaux, une chaudière de cuivre et différentes choses. Le $7^{\mathrm{e}}$, la veille de la foire de Crête, près Thonon, ils s'emparèrent de la balle d'un mercier. Elle était remplie de mouchoirs, de tabatières, de boucles et ils la vendirent au nommé Pierre Moichon, [16] demeurant rue Vallon à Thonon, et en reçurent 4 louis en payement. Le $8^{\mathrm{e}}$ vol fut fait dans un autre endroit, où ils prirent six mesures de farine, dont ils firent des matefaims ${ }^{81}$, aux prisons. Lorsque Rosay vendit le poisson aux Minimes ${ }^{82}$ il observa, dit-il, où était leur argent et le dit à ses camarades qui résolurent de l'enlever. Ce coup manqua, parce que lui, Rosay, ayant été mis en liberté, il n'osa l'entreprendre seul. Mais il projetait, avec Fornex, d'aller voler les Minimes et faire ce coup lorsqu'ils furent arrêtés. Rosay ajoute, avec naïveté : « si lorsque je fus mis dans ces prisons, l'on m'avait puni avec sévérité, peut-être me serais-je corrigé. Mais les crimes auxquels le séjour que j'y fis m'entraîna et la vie dissolue que nous y menions me corrompirent davantage encore. Et je finis par ne plus craindre les prisons et par croire que toutes ressemblaient à celles où j'étais ".

Interrogé sur les gens qui achètent des marchandises volées et qui reçoivent les voleurs chez eux, il a donné les noms et désignations suivantes.

Guérin d'Hauteville, marchand à Évian. Il achète tout ce qu'on lui apporte.

La femme de Jean Jacquier. Elle demeure près du bois de Blonay, au delà d'Évian.

Le fils de Claude Serre à Lugrin.

[17] Toute la famille Salignon à Évian. L'aubergiste est lui-même un voleur.

Destral et sa femme ${ }^{83}$, l'aubergiste, rue de la visitation à Thonon. Cette femme a été appelée pour une confrontation, et qui est jolie. Non seulement elle achète, mais elle indique où il y a des coups à faire. Son mari fait de même et recommande de prendre ce qu'il y a de mieux (ainsi qu'il est plus amplement détaillé page 20$)^{84}$.

La femme Destral avait précédemment acheté de la ratine, volée par Bruchon et Rosay à Rumilly, dans une boutique que leur avait indiquée le mari Destral qui est sergent, et qui avait fait un voyage à Chambéry pendant lequel il avait remarqué cette boutique à Rumilly.

Chapelle de Corsier a acheté d'eux des fromages et deux juments.

La sœur de la femme Simon, à Chêne sur Genève. Elle reçoit toutes espèces de marchandises.

À côté de chez la dite Simon, une grande femme gravée de petite vérole. Rosay y a vendu des effets et a vu que dans sa cave, elle faisait dénaturer des habits des soldats du Régiment de la République. 
L'Italien, qui tient la [tuilière] de Vessy. Il est fameux, dit Rosay. Il achète des chevaux et les [dépèce]. Il est à tout. Les voleurs trouvent chez lui un [abri] sûr.

L'aubergiste du Cerf à Carouge : c'est lui qui a acheté le vol fait au Plan-les-Ouates chez Mr. Picot.

[18]Thevenot et sa femme à Carouge. Ils vendent du vin et logent au-dessus du Lion d'Or.

Le Tambour ci-devant du [Roi] de Sardaigne à Carouge; il tient le Coq d'Inde.

À Genève. la Fripière dont on a parlé ci-devant.

Samuel Friech, cordonnier du Petit Saconnex. Il a ci-devant demeuré à Bellevue. Il est lié avec et loge souvent Lorrain La Toccante.

Au Chalet à Gobet dans le Jura ut supra. L'homme s'appelle Jean François.

Jeannette à Lausanne, vendeuse de vin près des boucheries. Ticon et Fornay lui vendirent des effets volés dans une boutique à Vevey au Bourg au Faure. Parmi ces effets, il y avait des mouchoirs de soie.

Le vieux Piccard à Douvaine. Il fait, de plus, des certificats pour les mendiants et a un cachet de la grandeur d'un louis d'or, qu'il appose dessus.

Rosay ayant appris que Bassière était arrêté, s'est écrié : « Tenez-le bien, c'est un fier coquin. Il y a bien des années qu'il ne vit que de vols. Il a commis de gros crimes, il sait faire les tournantes (fausses clefs). Il les fait chez le Tambour à Carouge, au Coq, ainsi qu'au Cerf. Je l'y ai vu y travailler. J'étais même présent lorsqu'il s'est tiré un coup de pistolet à la mâchoire.»

Il a parlé du muet et a dit : « Cet homme est très intelligent, il est l'associé de Jean le Blondin, l'un des plus adroits voleurs qui soit aux environs. Il l'accompagne toujours [19] et lui sert à porter les outils pour commettre les vols et ensuite les effets volés. » Il a ajouté que le dit muet est d'Anthy, du côté de Coudrée, en Chablais.

Ayant vu le coutre ${ }^{85}$ de charrue qui a servi à Bassière pour voler la fripière Jacques.

Rosay l'a reconnu pour l'un de ceux qui étaient dans le repaire dont il a été parlé. « Nous en avions trois, a-t-il dit, celui-là en est. Il a été volé à Ville-la-Grande. " (D'après ${ }^{86}$ cela, il paraît que Bassière a été joint à la bande d'Orléans depuis la détention de Rosay. Le premier rapport paraît confirmé par le muet qui a donné à entendre que le coutre avait été apporté du côté de Chêne, la clef neuve ajustée dans la maison ayant pour enseigne le Coq à Carouge et les autres clefs prises sur les tranchées, où elles étaient enterrées.)

Rosay a dit qu'il y avait un serrurier en ville, ouvrier dans une boutique de serrurier à la rue du Temple, lequel a travaillé ci-devant à Pessey et est fils d'un serrurier de Chêne sur Savoie, qui est surnommé Lucifer, qui travaille parfaitement les fausses clefs, de manière qu'elles montrent exactement les endroits où les [incontrures] ${ }^{87}$ des serrures gênent. Il les fait bien supérieures à celles de Bassière qui ne les sait faire qu'à crochets. Rosay a bu souvent avec lui en ville, et à Bellevue où il s'était engagé avec lui et Du Fornay. Tous les trois s'en allèrent, depuis le fort de l'Écluse, et revinrent. Lucifer avait toujours sur lui cinq ou six fausses clefs tournantes, de celles dont les montures s'ôtaient et se remettaient, ainsi que les pannetons. "C'est un gueux qui travaille peu et qui est comme moi », dit Rosay.

[20] Rosay a dit, qu'étant allé à Carouge, en juin 1786 avec Faucoup, matelassier au dit Carouge, ils y avaient trouvé Garnier qui dit avoir travaillé aux Eaux-Vives et connaître un pré où il y avait des indiennes et point de chien. Ils s'y rendirent tous les trois (sans 
Lafond qui n'avait point été de ce coup). Garnier tira deux pièces du côté de la haie, Rosay une du côté du chemin et Faucoup resta sur le chemin à les attendre. Ils les emportèrent. En chemin, Garnier vola deux poules chez Rey à Vésenaz. Ils allèrent ensuite à Douvaine, puis à St-Cergue où ils vendirent les indiennes, puis à Jussy où Rosay et Garnier partagèrent entre eux six écusneufs, produit de la vente des indiennes, et donnèrent trente sols à Faucoup qui est un Français. Il a soutenu, jusqu'au dernier moment, que Garnier était l'indicateur du vol fait chez le sieur Favre ${ }^{88}$ et qu'il avait tiré deux pièces, pendant que lui, Rosay, tirait l'autre.

Fortement exhorté par Messieurs les pasteurs ${ }^{89}$ et par son confesseur ${ }^{90}$ à dire la vérité, Rosay est convenu d'avoir commis le vol d'indiennes fait le 8 octobre de l'année dernière. Il dit l'avoir commis avec Joseph Antogniate, en passant par-dessus le mur de Mont-Choisy. Ils le portèrent chez Destral à Thonon qui leur reprocha de n'avoir pas bien choisi les pièces, leur disant qu'il fallait y retourner et prendre des indiennes à dessins pour des gilets. Il ajouta, qu'en conséquence, ils revinrent à Mont-Choisy et y commirent le vol que le fabriquant essuya le 17 du même mois, et qu'ils le vendirent à la femme Destral, observant que le mari les avait accompagnés, du côté de cette [21] ville, lorsqu'ils vinrent commettre le premier vol, c'est-à-dire la nuit du 8 oct[obre] 1786.

Rosay a parlé du fils Estrasiat. Il a dit qu'il a dépouillé une boutique à Aiguebelle, qu'elle était placée sous la halle et appartenait à un marchand espagnol, qu'il a tout emporté.

Il a avoué avoir fait un vol à Lugrin, au nommé Pierre Levray. Il était avec le petit $D u$ Fornay, celui qui a été dernièrement dans les prisons et qui mendie habituellement au bout du pont d'Arve. Il dit : " Nous entrâmes par une fenêtre. Je soupçonnais qu'il y avait de l'argent. Je cherchai avec soin et je trouvai dans un sac de châtaignes, un petit pot renfermant 50 livres, que nous emportâmes. "

Pressé et exhorté fortement à dire enfin la vérité, Rosay a enfin avoué l'assassinat de Musca en disant : «L'on avait emporté le magot du repaire. Nous fûmes persuadés que c'était Musca et qu'il l'avait caché quelque part avant d'entrer aux prisons à Thonon. C'est pourquoi, nous guettâmes le moment de sa sortie des dites prisons, pour lui faire restituer notre part de cet argent. Musca, que nous avions quitté la vieille au soir à Douvaine et rejoint le matin près de Corsier, et avec lequel nous avions déjeuné chez Chapelle et bu depuis lors à Veigy, ne disconvint pas d'avoir l'argent. Il nous proposa de le suivre, disant qu'il nous conduirait au lieu où était le magot. Nous fûmes dans les bois de Marnaud. [22]Musca déterra un mouchoir dans lequel était, outre l'argent susdit, deux montres d'argent et des boucles du même métal qui avaient été volées par Musca à des Genevois à Douvaine. Jospeh voulait sa part. Musca ne voulait pas la lui donner. Ils se prirent aux cheveux. Je les séparai. Ils se raccommodèrent et jouèrent même ensemble. Musca proposait d'aller dans la Suisse italienne. Joseph insistait pour avoir de l'argent. Il prit le moment où Musca, cueillant des champignons, ne le voyait pas, pour me faire signe de le frapper. Alors j'assénai un coup de mon bâton, sur la tête du dit Musca, qui le coucha par terre et je sautai sur le mouchoir qui contenait le magot. Je l'emportai et m'enfuis sur le Coteau de Boisy où Joseph me joignit. C'était à l'entrée de la nuit. Ce fut avec le bout du fléau, pris par Joseph la veille à Bonatré, que je frappai Musca. Je le jetai ensuite au loin dans le bois. Les deux autres bâtons trouvés près du cadavre étaient bien aussi les nôtres et l'un de ces deux bâtons, qui est court et droit, était celui que Joseph portait ordinairement, tandis que j'avais celui à [maillèche $]^{91}$. Il y avait environ deux mille livres dans le mouchoir, le tout en écus de six livres. Les pistoles ne se trouvèrent point dans le paquet. Je crois que Joseph s'en était saisi à la dérobée. 
Cet argent provenait, pour [23] la plus grande partie, du vol fait au curé de Thonex. Joseph me joignit sur le Coteau de Boisy [assez tard]. Nous prîmes la route du Valais, allant à SaintMaurice. Nous y arrivâmes le lendemain dimanche, à 8 heures du matin. Ceux qui ont dit que nous avions traversé le lac se sont bien trompés. Joseph, m'ayant donné 18 livres, pour payer notre dépense, il saisit le moment pour décamper avec l'argent. Depuis lors, je ne l'ai pas revu. La somme était dans un sac à poudre de peau que nous avions acheté pour la mettre. »

Il a dit que ceux qui avaient vu de l'argent à Fornay et à lui ne s'étaient pas trompés. Qu'ils avaient vingt-deux écus neufs volés au regrattier de Marin. C'est Duret ${ }^{92}$ qui les a dénoncés. Rosay à ajouté : «Les juges m'en ont bien parlé, mais comme ils ne faisaient mention que de 14 écus, je compris bien qu'ils n'avaient pas reçu la plainte du regrattier ${ }^{93}$ et qu'ils ne connaissaient ce vol que par des rapports. "

On a nommé devant lui un coquin connu sous le nom de Sans-Regrets. Rosay a dit : " C'est un des fameux. J'ai fait un fier coup avec lui. Nous étions l'été dernier dans un cabaret à Lully, au-dessus de Thonon. J'eus une dispute avec le cabaretier et trois autres hommes. C'était une chose à voir. Je pris le cabaretier et le précipitai dans le feu. La tête partit dans le pot de la soupe. Pendant ce temps-là, Sans-Regrets [24] et un autre volèrent de la toile que nous portâmes à Douvaine, chez la cabaretière qui l'acheta. "

Il a avoué, en outre, d'autres vols commis pendant qu'il était dans les prisons de Thonon. Celui d'une quantité de paires de souliers volées à un marchand étranger, lesquelles furent portées dans les prisons, à l'exception d'une paire qu'il mit à ses pieds.

Il a dit qu'il avait eu longtemps pour maîtresse la nommée Gillet, fille de celle qui resta, pendant quelques mois, à la maison de correction, comme suspecte de relation avec la bande de voleurs découverte à la fin de 1783. « Elle est ajoute-t-il, catin et voleuse. » Ayant su qu'elle avait donné un rendez-vous à un soldat, de Carouge, il les guetta et rossa tellement le soldat qu'il le laissa pour mort et jeta une pierre à la Gillet qui se sauvait. Cette pierre l'atteignit si bien qu'elle en fut renversée. Il parle de cette fille comme d'une coquine très dangereuse. Repris quelques moments après et pressé de nouveau de dire la vérité, Rosay a dit que, passant à Chesne avec Du Fornay le 20 ou le 22 juillet de l'année dernière, ils virent sur le banc de Chapalay ${ }^{94}$ un contrebandier qui dormait, ayant un ballot de sel à côté de lui. Qu'il lui volèrent son ballot, le portèrent [25] à [Meinier] ${ }^{95}$ et le vendirent pour 4 sols la livre aux gens des environs. "Ce ballot, dit-il, pesait plus de 100 livres. »

Il a encore avoué que l'automne dernier, étant avec Joseph sur le chemin de Chêne, ils arrêtèrent un paysan qui avait, dans une hotte, du pain et du fromage, qu'ils les lui prirent ainsi [qu'un] demi-écu qu'il avait dans sa poche. Qu'ils se tenaient à côté du petit chemin qui passe au travers des prés, à côté de Grange-Bonnet, dans l'intention, dit Rosay, de faire contribuer quelque passant qui put leur fournir assez d'argent. Joseph voulait qu'on le tua pour que ce fut plus tôt fait. Rosay assure qu'il s'y opposa toujours et que n'ayant point d'argent, la misère seul lui faisait faire ce métier. Pressé de dire s'ils n'attaquèrent personne ce jour-là, il a toujours assuré qu'ils ne s'adressèrent à personne, ne voulant le faire qu'à quelqu'un d'opulent. Il a ajouté encore, que, s'il avait nommé Orléans comme chef de la bande, c'est qu'il n'avait pas voulu parler de Musca, mais que c'était lui qui en était le chef lorsqu'il y fut conduit la première fois. Que quelques jours avant le vol que Musca fit à Douvaine, de deux montres et d'une paire de boucles d'argent, il emporta l'argent appartenant à la bande et fut enterrer le tout dans 
les bois de Marnaud et que ce ne fut [26] qu'alors qu'Orléans fut déclaré le Capitaine de la bande.

Rosay a fini en disant : " J'ai fait bien des vols dont je ne puis me rappeler. Je me suis servi quelquefois de mon bâton, soit dans les grands chemins, soit dans les cabarets, mais jamais je n'ai tiré mon couteau contre personne, ni n'ai volé ceux que j'ai attaqués sur le chemin. »

Du 14 juin 1787.

Nous, ancien Syndic, Conseiller et Auditeurs soussignés, certifions qu'ayant rassemblé tous les aveux que FrançoisRosay avait fait partiellement depuis quelques jours, nous les avons couchés par écrit comme dessus et que, ce aujourd'hui, après lui avoir annoncé que la grâce qu'il avait demandée au Grand Conseil ne lui avait point été accordée et qu'il ne lui restait que peu de temps à vivre, nous l'avons exhorté à l'employer à soulager sa conscience du poids de ses crimes, en nous mettant à même d'en recevoir une déclaration officielle et, particulièrement, de ceux qui avaient échappé à l'œil de ses juges. Ce à quoi l'ayant trouvé disposé, nous lui avons lu l'état de ses aveux, qu'il a confirmés après quelques changements à plusieurs d'entre eux. Déclarant que, couché comme il l'est ci-joint, il contient la vérité, témoignant du regret de ne les avoir pas fait connaître plus tôt et paraissant pénétré [27] de la justice du châtiment qui lui était infligé. En foi de quoi, nous dressons et signons le présent verbal.

De la Rive, cons[eiller], Thelluson Au[dit]eur, Saladin Aud[iteur].

\section{NOTES}

1. Ce travail résume un mémoire de recherche en Histoire moderne réalisé en 2003 à l'Université de Genève sous la direction de Michel Porret.

2. Egmond (1993).

3. Selon Ferrière, « [le testament de mort] ne sert à l'égard des complices que d'indice contre eux à effet de les faire emprisonner. Cependant, deux testaments de mort conformes contre une même personne forment un soupçon violent et peuvent, suivant les circonstances, suffire pour faire appliquer à la question ». Cf. Ferrière (1739, « testament de mort »).

4. Il n'existe qu'une seule étude systématique consacrée aux testaments de mort, $c f$. Crépin (1992). Par ailleurs, deux historiennes ont édité des «confessions» de criminels faites au Parlement de Paris entre 1329 et 1350. Cf. Langlois, Lanhers (1971).

5. AEG P[rocès] C[riminels] 1ère série, 15312-15313, désormais PC Rosay. Le dossier du procès compte 657 folios y compris les «Conclusions du Procureur général » et le « Testament de mort », ainsi qu'un tableau des « charges et des délits dont il est fait mention dans la procédure instruite contre François Rosay de Lugrin ». Nous proposons, en fin d'article, l'édition du testament de mort de Rosay.

6. Sur la justice genevoise, voir Porret (1995 a). Sur le banditisme genevois, voir Dubied (2001).

7. Briegel (2000). La plaidoirie de l'avocat de Rosay (Me De Rochemont) a disparu.

8. AEG PC (I) 14922.

9. Pour la « biographie criminelle», voir Porret (1995 b).

10. PC Rosay, $n^{\circ} 138$, Conclusions du procureur général.

11. AEG Jur. Pen. $\mathrm{H} 2 \mathrm{n}^{\circ} 2$ « Registre de signalements et de visites faites à Genève de condamnés, de prisonniers et de suspects (1775-1793)».

12. AEG, Archives de famille, Naville, XIV.A - 6/2 « Notes extraites d'un livre de notes de Mr P. Picot, pasteur et professeur ». Maintes fois, le pasteur Picot visite François Rosay en prison. 
13. $\mathrm{PC}$ Rosay, $\mathrm{n}^{\circ} 48$.

14. Précis historique de la vie de Coponex [Copponnais, Copponex], Lyon, 1764.

15. PC Rosay, $\mathrm{n}^{\circ} 38$.

16. ADHS 5Mi $932 n^{\circ} 9$ Registre des baptêmes, 1739-1751 et $n^{\circ} 11$ Registre des baptêmes, 1751-1793.

17. $\mathrm{PC}$ Rosay, $\mathrm{n}^{\circ} 138$, Conclusions du procureur général.

18. Trois cabaretiers receleurs selon le testament: Destral à Thonon, Chapelle à Corsier et l'aubergiste du Cerf à Carouge.

19. PC Rosay, $\mathrm{n}^{\circ} 15,16$ et 49 .

20. Dubied (2001)

21. Idem, p. 8.

22. Voir à ce sujet Chagniot (1985) et Corvisier (1992).

23. PC Rosay, $\mathrm{n}^{\circ} 3$, 31, et 58 .

24. Idem, $\mathrm{n}^{\circ} 138$, Conclusions du procureur général.

25. Idem, $\mathrm{n}^{\circ} 31$.

26. $\mathrm{PC}$ Rosay, $\mathrm{n}^{\circ} 25$, répétitions de Rosay.

27. Idem, $\mathrm{n}^{\circ} 138$, Conclusions du procureur général.

28. Voir ci-après le testament de mort, pp. 14-16.

29. Deyon (1975, p. 32)

30. Voir à ce sujet Bée (1983), Foucault (1975), Sharpe (1985).

31. Porret (1999, p. 397).

32. AEG RC 274 « Registre du Conseil », 1773, fol. 702, « Mémoire des pasteurs consolateurs » du 20 décembre 1773.

33. À partir d'un principe essentiel de la procédure inquisitoire, c'est-à-dire qu'il revient au juge de prouver la culpabilité d'un prévenu, la doctrine a élaboré une théorie de la preuve pénale qui n'admet comme preuves que l'aveu du prévenu ou les témoignages concordants. Voir Carbasse (2000, p. 167).

34. AEG, Cp. Past. R34, « Registre de la vénérable compagnie des pasteurs », p. 50.

35. AEG, RC 290-291, « Registre du Conseil » (1786-1787).

36. PC Rosay, $\mathrm{n}^{\circ} 138$, Conclusions du procureur général. Cette phrase centrale contient toute l'idée de la construction de la biographie criminelle.

37. AEG PC (I) 15312 et 15313 , pièce $n^{\circ} 144$.

38. 1 mètre 87.

39. Inconnu : cratère, trou, dépression?

40. Imprécision chronologique ? Selon la procédure, il semble que Rosay ait servi avec les troupes du Piémont après être revenu du régiment de Courten.

41. Régiment suisse au service de la France.

42. Inconnu.

43. Monia. Le village de Meinier est distant d'environ trois kms de l'Hermance.

44. 1.50 x 2.50 mètres.

45. Chapelle, cabaretier à Corsier, est entendu à titre de témoin dans le cadre de la procédure contre Rosay.

46. Les Ezolas.

47. Les recherches effectuées dès le mois de juillet 1787 dans le but de trouver le repaire n'ont rien donné. Il semble également que la bande dont parle Rosay ait été dispersée dès avant l'hiver 1786/1787. PC (I) 15030, lettre de l'intendant de Carouge du 11 juillet 1787.

48. Payer sur le pied : à raison de $7 \frac{1}{2}$ sous de Genève la livre.

49. Serre, cabaretier à Pressy, témoin dans la procédure contre Rosay.

50. $1.89 \mathrm{~m}$.

51. Assassiné par Rosay et Joseph Fornex. 
52. Selon une lettre de l'intendant de Carouge aux juges de Genève, Bougeon du Carre est détenu en Savoie suite aux aveux de Rosay. PC (I) 15030.

53. Le curé concerné a déposé devant les juges à Carouge. Selon Monnet de Thonex qui, chargé de s'informer, écrit à Genève, il n'y a « rien de si vague que le peu que sa mémoire lui rappelle sur ce sujet ». Le curé convient bien d'un vol, mais ne se souvient pas du montant. PC (I) 15303.

54. Dans le texte : montans de clefs vuides.

55. Partie de la clef qui pénètre dans la serrure et agit sur le pêne.

56. Jean-Baptiste Lhuillier, dit Lorrain la Toccante, de Valfroicourt en Lorraine, orfèvre et âgé de 35 ans arrêté le 24 octobre 1787 à Genève. La procédure engagée contre lui est fortement fondée sur les aveux de Rosay que les magistrats peinent grandement à prouver. Lorrain nie l'ensemble des accusations. Prisons subies et relâché faute de preuve, banni de Genève, le 22 février 1788. PC (I) 15303

57. Les recherches entreprises dans le cadre de la procédure engagée contre Lorrain disent d'elle qu'elle « est pauvre et semble innocente ». PC (I) 15303.

58. Jeanne Du Fornay, 20 ans et revendeuse en fruits, arrêtée le 27 octobre 1787 pour rupture de ban et dans le cadre de la procédure engagée contre Lorrain dit la Toccante, parce que suspecte, grâce aux aveux de Rosay, d'" être en relation avec Lorrain » et dépositaire de son argent. Elle est relâchée, bannie de Genève, le 3 novembre. PC (I) 15305.

59. Joseph Fornex, environ 25 ans en 1786, laboureur. Il est arrêté en même temps que Rosay en novembre 1786 et est prévenu d'être associé au vol des indiennes. Il comparaît le 15 décembre de la même année devant Sarasin et est relâché faute de preuve. Après 40 jours de prison, il est libéré et banni de la ville.

60. Selon une lettre de l'intendant de Carouge, Du Fornay, est détenu en Savoie à la suite des aveux de Rosay. PC (I) 15030.

61. Sollicité par les magistrats genevois, De Malle de Vevey dit à propos du vol du 28 juillet n'avoir « absolument rien découvert ». PC (I) 15303.

62. $1.65 \mathrm{~m}$.

63. Les jugements par contumace condamnent les prévenus qui sont en fuite. La peine qui en découle est exécutée en effigie. On expose ainsi au public une représentation symbolique du coupable et de la peine à laquelle il a été condamné.

64. Environ $1.71 \mathrm{~m}$.

65. Environ $1.65 \mathrm{~m}$.

66. Environ $1.68 \mathrm{~m}$.

67. Bassière dit Courtebotte est arrêté à Genève en avril 1787 et condamné en août de la même année à être « lié et garrotté, battu de verges dans tous les carrefours jusqu'à effusion du sang et banni à perpétuité » de la ville et des territoires de Genève. PC (I) 15159.

68. En août 1787, le petit conseil est prié par Mr Despine de bien vouloir faire les démarches pour « découvrir et arrêter si possible un scélérat » dont il donne le signalement. Il se pourrait que cet homme soit Joseph Antogniate, complice de Rosay. Registre du conseil, RC 291 p. 638.

69. Référence aux trois vols d'indiennes aux Eaux-Vives qui sont à l'origine de l'information engagée contre François Rosay. Ces trois vols constituent les délits importants effectués par Rosay sur le territoire de la République.

70. Vingy.

71. Inconnu.

72. La marque, pratiquée par la justice d'Ancien Régime dans la chair des coupables, est une trace indélébile signalant l'infamie. Il existe de nombreux types, allant $\mathrm{du}$ « V » marqué au fer rouge à l'essorillement.

73. D'après les courriers échangés entre les magistrats genevois et les maires du Locle et de La Chaux-de-Fonds, il semble qu'il n'existe aucune trace de ce vol dans les montagnes neuchâteloises. Selon Robert de La Chaux-de-Fonds, « il est très certain qu'il ne s'est point fait de 
vol de vaisselle d'argent à La Chaux-de-Fonds en 1785, ni dans aucun autre temps de mon souvenir, et le nom de Rillaud m'est absolument inconnu ainsi qu'à nombre de personnes à qui j'ai parlé de cette affaire ". Selon lui, il est possible que ce vol de vaisselle ait été fait à Morteau et que Rosay se soit trompé. PC (I) 15303 , pièce $n^{\circ} 6$.

74. Replacée dans son contexte judiciaire et son époque, cette phrase, d'apparence anodine, est une véritable dénonciation, puisqu'il est interdit de donner refuge ou de faire du commerce avec les brigands et vagabonds douteux. Ce cas-ci, un couple en "grande relation " avec un brigand est un exemple intéressant de recel (achat à bas prix, transformation et revente à prix fort de marchandises volées). Voir aussi pp. 16 et suivantes du testament.

75. Faire disparaître, cacher. Ici : voler.

76. Du Malle de Vevey écrit le 30 novembre 1787 à Genève l'état de la situation : « [...] tel est l'état $\mathrm{du}$ local [du Sieur Collomb] qui aurait rendu bien difficile l'entreprise de Lorrain. [...] aucun verbal n'a été fait à l'occasion de vol de montres ». PC (I) 15303.

77. Trafiquant qui spécule de manière illicite sur les cours des monnaies ou autres valeurs.

78. En juillet 1786, Rosay est arrêté à Genève pour « effraction de pavillon » et ensuite banni de la République. PC (I) 14922.

79. Inconnu.

80. Inconnu.

81. Galette épaisse.

82. Ordre religieux mendiant fondé par St-François de Paule.

83. Les époux Destral, témoins dans le procédure contre Rosay, sont arrêtés et détenus en Savoie à la suite des aveux de Rosay. Ils y sont prévenus d'être impliqués en qualité de receleurs dans un des vols d'indiennes. PC (I) 15030.

84. Rajout, même écriture que dans la marge.

85. Fer tranchant fixé à l'avant du soc de la charrue pour fendre la terre.

86. En marge : Le paragraphe dès le mot «d'après » à la fin est une observation et non une confession de Rosay.

87. Inconnu.

88. La fonction de Garnier dans ce vol mérite d'être relevée. Il semble en effet qu'il ait fonctionné comme indicateur, c'est-à-dire comme la personne qui connaît les lieux et l'intérêt qu'ils ont. Le fait est que Garnier a travaillé chez le fabriquant d'indiennes, ce qui permet au procureur général Naville de qualifier la participation de Garnier au vol d'indiennes de "vol domestique ", circonstance aggravante.

89. Les pasteurs Thouron et Peschier Le Jeune ont accompagné Rosay au supplice. Registre de la Compagnie des pasteurs, année 1787.

90. Le capucin Père Édouard du couvent de Gex. PC (1) 15030, «Verbal de De la Rive », 4 août 1787.

91. Inconnu.

92. Duret dépose dans la procédure Rosay. Son témoignage est intéressant puisqu'il touche l'engagement de Rosay dans le régiment de Courten et sa désertion. Duret, après avoir déserté aux côtés de Rosay, s'est engagé au Régiment de la République. PC (I) 15312.

93. Personne qui fait le commerce de regrat, soit la vente de denrées au détail et de seconde main.

94. Inconnu.

95. Meny. 


\section{RÉSUMÉS}

Basé sur le seul procès criminel de François Rosay, cet article propose un éclairage particulier sur un type de documents étonnants, les « testaments de mort ». L'intérêt de ces textes réside dans le fait qu'il s'agit de la mise en écrit par des magistrats d'aveux faits non pas au cours d'un procès mais juste avant l'exécution de la sentence, au pied du gibet. La lecture du testament de mort de Rosay et du très complet procès qui l'accompagne permet de porter quelques constatations sur la biographie criminelle du brigand et sur son environnement social ainsi que de revenir sur la nature de la criminalité associative. Le récit des aveux de Rosay, sous sa forme de «testament de mort ", est intégralement publié en deuxième partie.

This article presents an analysis of François Rosay's, «last dying speech ». The interest of this specific type of document, lies in the fact that it presents the transcription by judges of confessions which have not been made during a trial, but on the gallows, just before the execution. From an examination of Rosay's speech and very complete trial record, the authors draws some conclusions regarding this criminal's biography and environment, and also the nature of banditry. Rosay's complete confession is appended to the article.

\section{AUTEUR}

\section{EMANUEL GOGNIAT}

Haut du village, 24, CH-2718 Lajoux, emanuel.gogniat@bluewin.ch

Emanuel Gogniat, spécialisé en histoire contemporaine et moderne, est licencié ès Lettres de l'Université de Genève (2003). Il travaille actuellement dans l'enseignement et prépare la publication de son mémoire de licence consacré à la Question jurassienne. Il est également l'auteur de Aux racines du patriotisme : affaire de la place d'armes des Franches-Montagnes et Question jurassienne (1956-1976), Courrendlin (CH), Éditions communication jurassienne et européenne (2005, sous presse). 